\title{
Nuclear magnetic resonance evaluation of inflammatory activity from chronic viral Hepatitis B
}

\author{
Jinghui Dong', Yuan Liu², Huiyi Ye3, \\ Weimin $\mathrm{An}^{4}$, Hongwei Ren
}

\begin{abstract}
Objectives: To discuss the value of applying magnetic resonance diffusion-weighted imaging (DWI) to evaluate inflammatory activity from chronic viral hepatitis B.

Methods: One hundred forty-two patients with chronic viral hepatitis B who received treatment at The Fifth Medical Center of Chinese PLA General Hospital from January 2014 to December 2015 and 20 healthy persons in the control group who were scheduled to undergo nuclear magnetic resonance scanning and DWI examinations ( $b$ value $=0,800 \mathrm{~s} / \mathrm{mm}^{2}$ ), and the apparent diffusion coefficients (ADCs) were measured and compared with the biopsy results of hepatic tissue.

Results: The ADC value of the group with hepatitis B was lower than that of the healthy group $(P<0.05)$, and the $A D C$ value of the group with mild inflammation (G1) significantly differed from that of the group with moderate inflammation (G2) and that of the group with severe inflammation (G3-G4) $(P<0.05)$.

Conclusions: Magnetic resonance diffusion-weighted imaging technology has high clinical value for evaluating the inflammatory activity from chronic hepatitis $B$, and the measured ADC value corresponds to the pathological grade well, so this method is worth clinical promotion and application.
\end{abstract}

KEYWORDS: Hepatitis B, Inflammatory activity, Magnetic resonance imaging, Diffusion-weighted imaging.

How to cite this:

doi: https://doi.org/10.12669/pjms.35.6.1364

Dong J, Liu Y, Ye H, An W, Ren H. Nuclear magnetic resonance evaluation of inflammatory activity from chronic viral Hepatitis B. Pak J Med Sci. 2019;35(6):1565-1569. doi: https://doi.org/10.12669/pjms.35.6.1364

This is an Open Access article distributed under the terms of the Creative Commons Attribution License (http://creativecommons.org/licenses/by/3.0), which permits unrestricted use, distribution, and reproduction in any medium, provided the original work is properly cited.

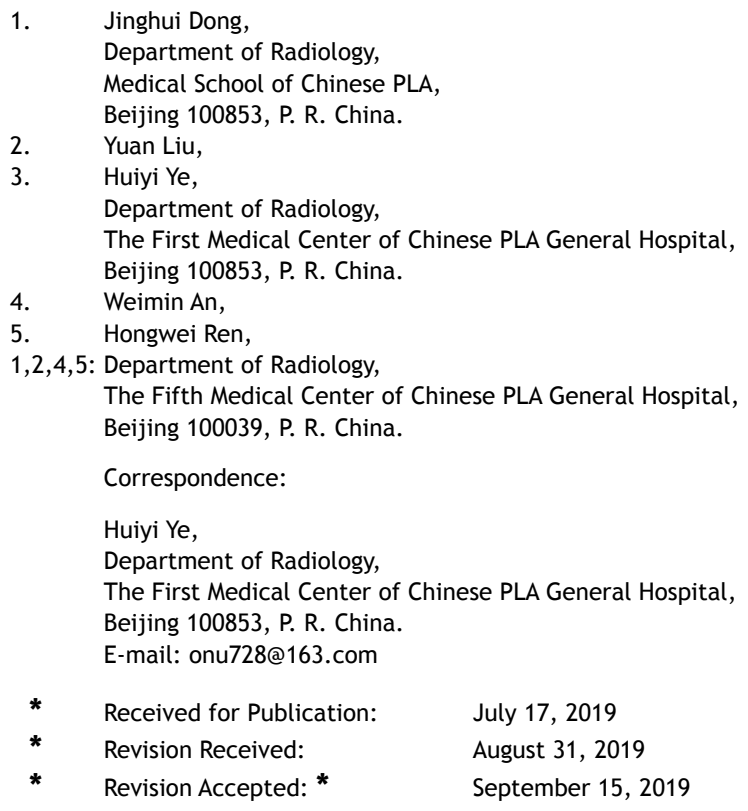

\section{INTRODUCTION}

In China, the number of carriers of hepatitis B accounts for ten percent of the total population. Chronic viral hepatitis is persistent and recurrent and ultimately develops into hepatic fibrosis, hepatic cirrhosis and even hepatic carcinoma, all of which gravely threaten patient health. ${ }^{1}$ Generally, lesions from chronic hepatitis B are associated with inflammation and fibrosis. The inflammation grade of hepatic tissue reflects the current status of liver injury and hepatitis activation. The hepatic fibrosis stage indicates cumulative liver injury and is related to the course of disease. If treatment is offered during this period, then the lesion can be reverted to healthy tissue. ${ }^{2}$

The current gold standard for evaluating the inflammation indexes of chronic hepatitis B is liver biopsy. However, liver biopsy is invasive and has various disadvantages, such as limited sampling, 
low patient compliance, and poor examination repeatability. Thus, a simple and noninvasive method with high repeatability is sorely needed to evaluate the inflammatory activity of chronic hepatitis B. Magnetic resonance diffusion-weighted imaging (DWI) technology has a clear advantage in diagnosing hepatic diseases. This study aimed to preliminarily discuss and compare magnetic resonance diffusion-weighted imaging and pathological grading in assessing inflammatory activity from chronic hepatitis B; DWI is hopefully of great clinical significance in guiding clinical diagnosis and treatment, therapeutic evaluations and follow-up visits for chronic hepatitis.

\section{METHODS}

One hundred forty-two patients with chronic viral hepatitis B admitted to The Fifth Medical Center of Chinese PLA General Hospital from January 2014 to December 2015 were observed, including 89 male patients and 53 female patients. The patients were aged 18 to 67 ( $42.5 \pm 5.8$ on average).

Ethical Approval: The study was approved by the Institutional Ethics Committee of The Fifth Medical Center of Chinese PLA General Hospital, and written informed consent was obtained from all participants.(Approval date December 8, 2013)

All the selected patients complied with the diagnostic criteria as specified in the 2005 Guideline on Prophylaxis and Treatment of Chronic Hepatitis $\mathrm{B},{ }^{3}$ were confirmed not to have hepatitis $\mathrm{B}$ with hepatitis C, hepatitis D, cytomegalovirus, human papillomavirus virus, HIV, alcoholic liver disease, drug-induced hepatitis, autoimmune liver disease, or cholestatic liver disease, and were not complicated with cirrhosis or liver tumors. The patients immediately underwent liver biopsy within two days after MRI, and two associate chief physicians from the pathology department jointly read the slices, consulted with each other and determined the histopathologic grade. The severity of hepatitis was graded as G0-4 and S0-4 in accordance with the standards revised at the $2000 \mathrm{Xi}^{\prime}$ an National Virus Hepatitis and Liver Disease Conference. ${ }^{4}$ Since the number of G4 cases among this group was small, G3 and G4 were combined as severe inflammation. On this basis, liver inflammation activity was classified as mild (G1), moderate (G2) and severe (G3, G4). Moreover, 20 healthy persons were selected as the control group, and these patients had no preexisting liver disease, had normal hepatic function indexes and were aged 30 to $56(46.1 \pm 3.2$ on average). All the selected patients were approved by the hospital ethics committee, and the patients were asked to sign informed consent Forms.

Examination Method: The selected patients received conventional MR scanning and DWI scanning. For the MRI examination, a GE HD x 3.0T high-intensity magnetic resonance imaging system and an 8 -channel phased-array body surface coil were adopted, and the scanned area was from the diaphragmatic dome to the inferior margin of the liver. DWI scanning was followed by axial T1WI and T2WI scanning. The scanning parameters were as follows: TR $1900 \mathrm{~ms}$, TE $64 \mathrm{~ms}, \mathrm{~b}=800 \mathrm{~mm}^{2} / \mathrm{s}$, slice thickness $8 \mathrm{~mm}$, interlayer spacing $1 \mathrm{~mm}$, view $38 \mathrm{~cm} \times 38 \mathrm{~cm}$, matrix 128x128, number of excitations 2 , number of scanning layers 20 , and scan time $29 \mathrm{~s}$; the diffusion direction was all directions, breathless collection was conducted on the end-expiratory row, and two breath holding cycles were conducted to complete scanning.

ADC Value Measurement: The ADC values of three regions of interest of the same size in different locations on each of the three consecutive layers of the right rear lobe of the liver were measured with the Function tool of the software in the AW4.5 workstation equipped with a GE HDxt3.0T MR machine. The area of the region of interest was approximately $200-300 \mathrm{~mm}^{2}$, and blood vessels, bile ducts, and chemical shift artifacts were avoided; the average of the nine values was taken as the observed ADC value of the liver.

Table-I: Criteria for staging and grading chronic hepatitis (2000, Xi'an).

\begin{tabular}{|c|c|c|c|c|}
\hline \multicolumn{2}{|c|}{ Inflammatory activity } & \multicolumn{3}{|l|}{ Degree of fibrosis } \\
\hline Grade & Portal area and periphery & Intralobular & Stage & Fibrosis \\
\hline G0 & No inflammation & No inflammation & so & None \\
\hline G1 & Portal area inflammation & Degeneration and a little focal necrosis & S1 & Portal area expansion, fibrosis \\
\hline G2 & Mild piecemeal necrosis & $\begin{array}{l}\text { Degeneration, spotted and focal } \\
\text { necrosis or acidophilic body }\end{array}$ & S2 & $\begin{array}{l}\text { Fibrous septum is forming, } \\
\text { and lobular structure is retained }\end{array}$ \\
\hline G3 & $\begin{array}{l}\text { Moderate piecemeal } \\
\text { necrosis }\end{array}$ & $\begin{array}{l}\text { Degeneration, severe necrosis or is } \\
\text { visible bridging necros }\end{array}$ & S3 & $\begin{array}{l}\text { Fiber insulation with lobular } \\
\text { disorder, without liver cirrhosis }\end{array}$ \\
\hline G4 & Severe piecemeal necrosis & $\begin{array}{l}\text { Wide area of bridging necrosis to } \\
\text { involve multiple lobules, lobular } \\
\text { structure disorder }\end{array}$ & S4 & Forepart hepatocirrhosis \\
\hline
\end{tabular}




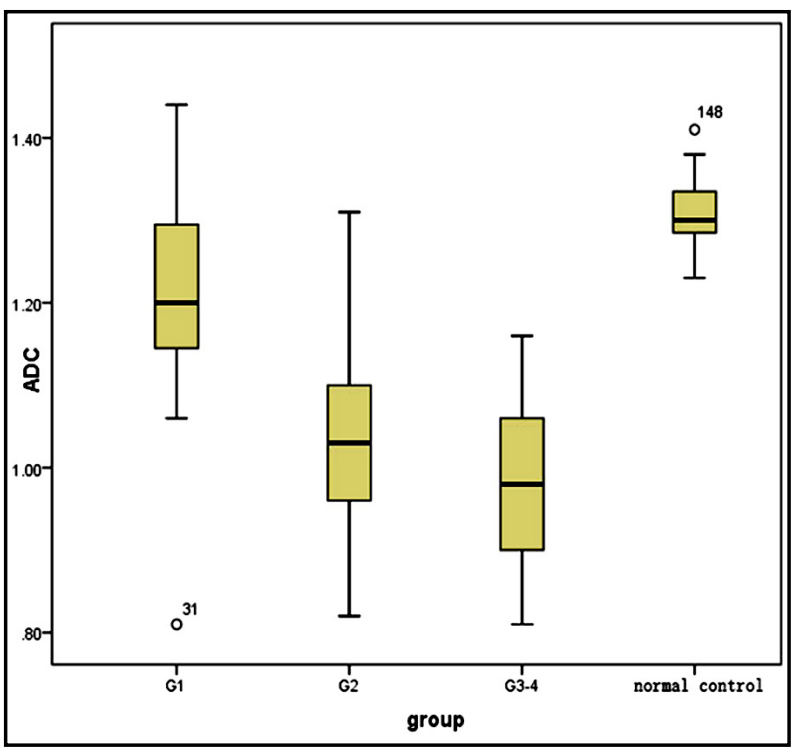

Fig.1: ADC values of groups with different grades of inflammation from Chronic Hepatitis B and the Normal Control Group (x10-3 mm2/s).

Grading Standard: ${ }^{4}$ The severity of hepatitis was graded and staged via the Knodell HAI score (Table-I).

SPSS 21.0 software was applied for data processing and analysis, and the mean \pm standard deviation $(X \pm s)$ was used to denote measurement data; variance analysis was adopted for intergroup comparisons, the LSD-q test was adopted for intragroup pairwise comparisons, and $\mathrm{P}<0.05$ indicated statistical significance. The receive operator characteristic (ROC) curve was used to evaluate the efficiency of diagnosing liver G2 or higher inflammation via the ADC value.

\section{RESULTS}

The box plots of the normal control group and different grades of inflammatory activity from chronic hepatitis $B$ when $b=800 \mathrm{~s} / \mathrm{mm}^{2}$ are shown in Fig.1. The ADC values of both the moderate (G2) and severe (G3, G4) inflammation groups were significantly lower than those of the mild (G1) inflammation and normal control groups (G0) $(\mathrm{P}<0.05)$ (Table-II).

Table-II: ADC values of the normal control group and groups with different grades of inflammation from Chronic Hepatitis B (x10-3 mm2/s).

\begin{tabular}{lcccc}
\hline & $\begin{array}{c}\text { Control } \\
\text { group }(\mathrm{G} 0)\end{array}$ & $\begin{array}{c}\text { Mild } \\
(\mathrm{G} 1)\end{array}$ & $\begin{array}{c}\text { Moderate } \\
(\mathrm{G} 2)\end{array}$ & $\begin{array}{c}\text { Severe } \\
(\mathrm{G} 3, \mathrm{G} 4)\end{array}$ \\
\hline $\mathrm{N}$ & 20 & 43 & 61 & 38 \\
$\begin{array}{c}\mathrm{ADC} \\
\text { value }\end{array}$ & $1.31 \pm 0.16$ & $1.22 \pm 0.12$ & $1.05 \pm 0.12^{*}$ & $0.98 \pm 0.10^{*}$ \\
\hline
\end{tabular}

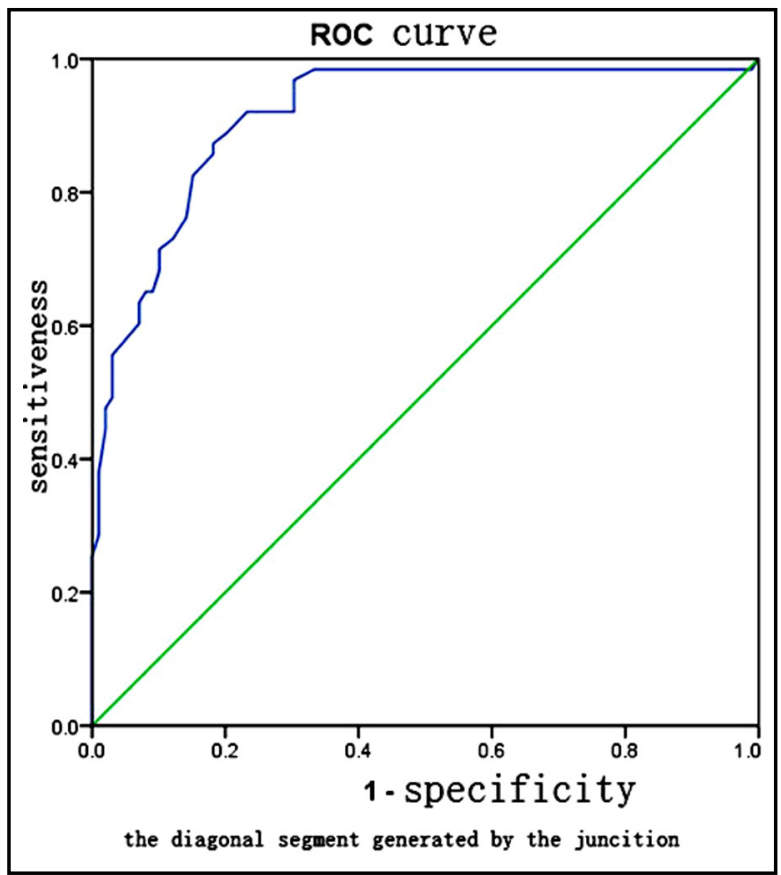

Fig.2: ROC Curve of G2 or Higher Inflammation Activity in Patients with Chronic Hepatitis B diagnosed via the ADC Value.

\section{DISCUSSION}

The overall objectives of treating patients with hepatitis B according to the Guideline on Prophylaxis and Treatment of Chronic Hepatitis B are to inhibit HBV for as long as possible, alleviate hepatocyte inflammatory necrosis and hepatic fibrosis, to delay and reduce liver decompensation, liver cirrhosis, hepatocellular carcinoma and complications thereof, to improve patient quality of life and to prolong survival time. Both domestically and internationally, it is universally considered that the key to delaying the progression of chronic hepatitis B is antiviral therapy. Sometimes, clinical and laboratory examinations are not reliable for guiding clinical treatment. Thus, pathological results are needed to guide clinic treatment, and it is noted that an ALT $\geq 2 \mathrm{ULN}$ or inflammation graded as G2 or higher and/or as S2 or higher is an indicator to initiate antiviral therapy. ${ }^{5}$ Patients with an ALT<2ULN usually have no obvious symptoms, which are often ignored by both doctors and the patients themselves. For such patients, antiviral therapy is recommended once liver inflammation reaches grade G2. According to previous studies, ${ }^{6,7}$ the proportion of patients with chronic hepatitis B with an ALT<2ULN suffering from obvious inflammation and hepatic fibrosis is large. Presently, liver biopsy is the gold standard 
for evaluating the inflammatory activity of chronic hepatitis B. However, liver biopsy is invasive, and the sampling coverage is limited. Thus, the degree of inflammation in the whole liver cannot be evaluated. In addition, patients with severe inflammation are not appropriate candidates to undergo liver biopsy. Thus, liver biopsy is not a good method for dynamically monitoring the progression of inflammation severity and evaluating curative effects. Therefore, a noninvasive method with high repeatability is needed to evaluate the severity of inflammation in the liver.

Early diagnosis of diffuse hepatopathy is of great significance for guiding clinical treatment and evaluating curative effects but is rarely detected with imaging, which is mainly applied for observing anatomical structure and morphological changes. ${ }^{8}$ In recent years, with the development of magnetic resonance technology, DWI has emerged as a noninvasive functional imaging technology to analyze the interior structures and tissue elements of lesions based on the diffusion motion of water molecules. The basic lesions of chronic hepatitis B feature inflammation and fibrosis. Pathologically, inflammation grade and fibrosis severity represent the current injury status of the liver and cumulative injury of inflammation, respectively, which are related to the duration of inflammatory injury. Inflammation of hepatic tissue will develop into hepatic fibrosis to a certain extent if immediate treatment is not conducted, and early hepatic fibrosis can recover with treatment. Therefore, the early diagnosis and treatment of this disease are of great importance. It was reported ${ }^{9}$ that inflammatory necrosis of hepatic tissue may result in an increase in portal vascular resistance, slow down the blood velocity of the portal vascular, and further cause hepatic fibrosis to develop into hepatic cirrhosis. Hepatic cirrhosis is almost irreversible. Therefore, the progression of chronic hepatitis B can be delayed by controlling changes in liver inflammation, which is of great significance for guiding subsequent clinical treatment and affecting the prognosis at follow-up.

The ADC value varies with the $b$ value. Thus, it is important to apply a proper $b$ value. The liver is a solid organ with rich blood circulation, so hemoperfusion will affect the accuracy of the ADC measurements. Studies have shown that a small $b$ value leads to a high image SNR and a clear image; however, hemoperfusion has a great impact on the ADC value because the diffusion motion of water molecules cannot be reflected well, so the measured ADC value is overestimated. The impact of hemoperfusion is negatively correlated with the $b$ value; $a$ large $b$ value will lead to a relatively accurate ADC value, ${ }^{10}$ but this relationship is not always the case. In the case of an excessively large $b$ value, the image SNR is drastically reduced, the artifact is large, and the measured ADC value is not accurate. ${ }^{11}$ To sum up, when $b=800$ $\mathrm{s} / \mathrm{mm}^{2}$, hemoperfusion has a slight impact, and the diffusion motion of water molecules can be truly reflected, which means that clear images are obtained, and the measured ADC value is stable.

The ADC value of the liver is determined by liver inflammation and fibrosis. Most previous reports did not include layering research on these two aspects, and the impact of hepatic fibrosis on the ADC value was not excluded, so the measured ADC value was relatively inaccurate. The studies by Ying et al. ${ }^{6}$ and $\mathrm{Li}$-tao $\mathrm{S}^{12}$ show that there exists a significant difference in ADC value between $\mathrm{S} 0$ and S3, S0 and S4, S1 and S3, and S1 and S4, while the difference among S0, S1 and S2 is not statistically significant. The fibrosis stages of the observed patients in this study ranged from S0 to S2, so S3 and S4 were excluded. In this way, the measured deviation caused by the impact of fibrosis on the $\mathrm{ADC}$ value of hepatic inflammatory activity can be avoided as much as possible. Moreover, the ADC value of the liver is affected by fatty infiltration and iron deposition. Since iron is a paramagnetic substance, iron deposition will shorten the T2 time of the liver to impact the quality of the DWI image and lead to inaccurate ADC values. Fatty infiltration causes the number of free protons in the liver to increase and affects the accuracy of the ADC value. For the purpose of this study, patients with fatty livers and iron overload were excluded so that the patients with chronic hepatitis B would pass the DWI evaluation of inflammatory severity and that this method provides quantitative reference indexes for clinical treatment and prognosis evaluations.

This study suggests that when $b=800 \mathrm{~s} / \mathrm{mm}^{2}$, the differences between mild (G1) and moderate (G2) inflammatory activity and between mild (G1) and severe (G3, G4) inflammatory activity are statistically significant. According to the therapeutic standards specified in the Guideline on Prophylaxis and Treatment of Chronic Hepatitis B, patients with G2 or higher inflammation should receive antiviral therapy. Thus, patients G2 or higher inflammation were taken as the inflammation group in this study, and those patients with G0-1 inflammation were taken as the non-inflammation group. According to the characteristics of the ROC curve (Fig.2), the 
area below the ROC curve reaches a maximum when $\mathrm{ADC}=1.13$, and a high accuracy is achieved when the AUC=0.914; the sensitivity and specificity of the inflammation group and non-inflammation group are $87.3 \%$ and $81.8 \%$, respectively. When the ADC is $>1.13$, patients are diagnosed with G0-1 inflammation. In such cases, no antiviral therapy is needed if the ALT is normal. When the ADC is $\leq 1.13$, patients are diagnosed with G2 or higher inflammation, and the patients should actively take antiviral therapy. In other words, an ADC $=1.13$ is regarded as the best critical value for initiating antiviral therapy.

Limitations of the study. First, there are differences between the liver biopsy method and the method for measuring ADC values. To address this problem, an ROI of the right rear lobe of the liver was selected to measure the ADC value to be as consistent as possible with the biopsy method. Second, a single b value was applied to research the degree of inflammatory necrosis of the liver to avoid balancing the impacts of hemoperfusion and diffusion on the ADC value. Thus, multiple $b$ values should be applied to ensure the accuracy of the measurement, which will be further discussed and studied in the future. Although DWI can be regarded as a quantitative analysis method, this study suggests that the differences in ADC value between the hepatitis group and normal control group and between the inflammation group and non-inflammation group are significant and can help make clinical antiviral therapeutic schemes, provided that the other clinical laboratory indexes are referred to.

In summary, magnetic resonance diffusionweighted imaging can reflect early diffuse lesions of the liver well, ADC measurements can help with the diagnosis, and dynamic observations of the ADC value can reveal the progression of inflammatory activity in the liver and the therapeutic effect; DWI has high application value in evaluating the inflammatory activity of patients with chronic hepatitis B and is worth clinical promotion and application.

Conflict of interests: None.

Funding: This study was supported by the Beijing Municipal Science \& Technology Commission (No. Z181100001718006).

\section{REFERENCES}

1. Sandrasegaran K, Tahir B, Patel A, Ramaswamy R, Bertrand K, Akisik FM, et al. The usefulness of diffusionweighted imaging in the characterization of liver lesions in patients with cirrhosis. Clin Radiol. 2013;68(7):708-715. doi: 10.1016/j.crad.2012.10.023

2. Xiao-jie Z. Diffusion-weighted magnetic resonance imaging in the diagnosis of hepatic fibrosis. Shanxi Med J. 2012;41(6):565-566. doi: 10.3969/j.issn.0253-9926.2012.06.021

3. Jun S, Yu-xin S, Zhi-yong Z, Zhan-qing Z, Jing-tao W, Jing Y. Clinical Study of the Correlation between MR ADC Value and Histopathologic Stage in Hepatic Fibrosis. J Pract Radiol. 2010;26(11):1609-1612. doi: 10.3969/j.issn.10021671.2010.11.018

4. Chinese Medical Association Infectious Diseases and Parasitic Diseases Branch, Liver Diseases Branch. Viral hepatitis prevention and treatment program. Chin J Hepatol. 2000;8(6):342-329. doi: 10.3760/j.issn:1007-3418.2000.06.001

5. Chinese Medical Association Liver Diseases Branch, Chinese Medical Association Infectious Diseases Branch. Guidelines for the Prevention and Treatment of Chronic Hepatitis B (2010 Edition). Chin J Virol. 2011;1(1):9-23.

6. Ying H, Lin S, Yan-min L, Hui-guo D, Shu-zhao W. Clinical significance of pathological examination to patients with chronic hepatitis B having normal or mild abnormal liver transaminases. Clin Focus. 2011;26(21):1871-1873.

7. Feng G, Xian-ni F. Clinical Observation on Serological Indicators, Pathological Grading and Staging of Chronic Hepatitis B with Alanine Transaminase Less than 2-fold Normal Upper Limit. Clin Focus. 2012;27(3):230-231.

8. Fallowfield J, Hayes P. Pathogenesis and treatment of hepatic fibrosis: is cirrhosis reversible? Clin Med (Lond). 2011;11(2):179-183.

9. Valla D, Flejou JF, Lebrec D, Bernuau J, Rueff B, Salzmann JL, et al. Portal hypertension and ascites in acute hepatitis: clinical, hemodynamic and histological correlations. Hepatology. 1989;10(4):482-487. doi: 10.1002/ hep.1840100414

10. Qiu-ju L, Jia-hui L, Zhou-she Z, Bing Y, Yu S, Qi-yong G. Assessment of aquaporins function of early-stage liver fibrosis using multi-b diffusion weighted magnetic resonance imaging. J China Clin Med Imaging. 2014;25(10):719-723.

11. Klasen J, Lanzman RS, Wittsack HJ, Kircheis G, Schek J, Quentin M, et al. Diffusion-weighted imaging (DWI) of the spleen in patients with liver cirrhosis and portal hypertension. Magn Reson Imaging. 2013;31(7):1092-1096. doi: 10.1016/j.mri.2013.01.003.

12. Li-tao S. Clinical Value of the Degree of Liver Fibrosis and Inlfammation of Hepatitis B by Magnetic Resonance Diffusion Weighted Imaging. Chinese J CT MRI. 2015;13(9):81-83. doi: 10.3969/j.issn.1672-5131.2015.09.025

\section{Author Contributions:}

JD \& YL: Collected and analyzed clinical data and prepared this manuscript, are responsible for integrity of research.

HY: Designed this study.

WA \& HR: Significantly revised this manuscript. 